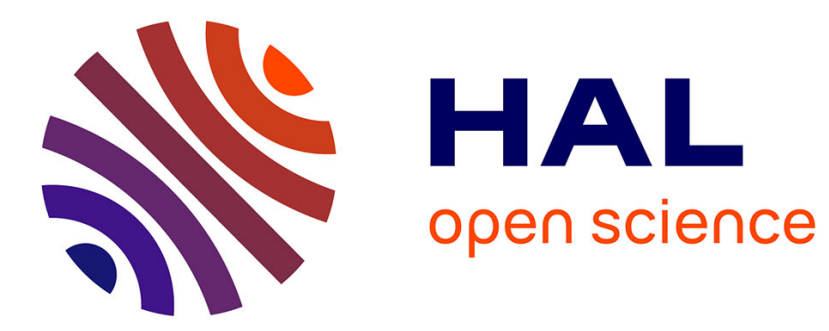

\title{
Utilisation et intérêt de la visite de pré-reprise : enquête réalisée auprès de médecins du travail
}

Sandra Pires, Florian Tone, Karine Wloch, Sophie Fantoni-Quinton

\section{To cite this version:}

Sandra Pires, Florian Tone, Karine Wloch, Sophie Fantoni-Quinton. Utilisation et intérêt de la visite de pré-reprise : enquête réalisée auprès de médecins du travail. Archives des Maladies Professionnelles et de L'Environnement, 2016, 77 (3), 10.1016/j.admp.2016.03.080 . hal-01721643

\section{HAL Id: hal-01721643 \\ https://hal.science/hal-01721643}

Submitted on 12 Mar 2018

HAL is a multi-disciplinary open access archive for the deposit and dissemination of scientific research documents, whether they are published or not. The documents may come from teaching and research institutions in France or abroad, or from public or private research centers.
L'archive ouverte pluridisciplinaire HAL, est destinée au dépôt et à la diffusion de documents scientifiques de niveau recherche, publiés ou non, émanant des établissements d'enseignement et de recherche français ou étrangers, des laboratoires publics ou privés. 
Utilisation et intérêt de la visite de préreprise :

Enquête réalisée auprès de médecins du travail

Use and interest of prereinstatement visit:

Survey conducted with occupational doctors

S. Pires, Médecin du Travail

F. Tone, Médecin du Travail, Pôle Santé Travail Métropole Nord

K. Wloch, Statisticienne, Pôle Santé Travail Métropole Nord

S. Fantoni Quinton, Professeur de médecine du travail/ Docteur en droit, CHRU Lille, UF

Pathologies professionnelles et Maintien dans l'emploi - Employabilité (Médecine du

travail), Université Lille2, Centre de Droit et Perspectives du droit, EA 4487

Enquête réalisée dans le Service Interentreprises de Santé au Travail «Pôle Santé Travail Métropole Nord », 199/201 Rue Colbert, Lille 
Purpose of the study: The preservation of employment is an overarching mission for the occupational health teams in France. The prereinstatement visit (VPR) is part of the steps to initiate early this purpose. The main objective of this work is to study the actual terms of implementation of the VPR.

Method: Prospective, descriptive study, in two steps. First step: based on a questionnaire, completed during the VPR, by doctors from an occupational health service, for a period of one month. Second step, six months after the VPR, become of these employees has been studied by a computer data analysis of the conclusion of the return to work visit (VR) if appropriate, and a phone contact.

Results: 172 VPR questionnaires were analyzed (48\% were women; mean age was 43.7 years; $56 \%$ were employees): $67 \%$ of employees were seen in VPR for the first time, $51 \%$ were off work for more than six months; VPR was at the initiative of employees in $58 \%$ of cases. After the VPR, a development of working conditions was advocated in $21 \%$ of cases and of the working time in $19 \%$ of cases. $50 \%$ of the 116 employees seen in VR benefited of a layout of their workstations, and 34\% were declared unemployable. The conclusion of the VR was significantly different depending on the existence or not of approaches advocated by the occupational doctors during the VPR $(\mathrm{p}=0.034)$, the contact with external stakeholders in the company $(\mathrm{p}=0.03)$, or with the employer $(\mathrm{p}=0.017)$, and depending on the type of disease $(\mathrm{p}=0.04)$. The becoming of the employees, collected by phone contact, was significantly different depending on the existence of a contact with the employer $(p=0,017)$; the other crossmatches were not significant.

Conclusion: The VPR is a key step in the process of return and keep to work, and impact the conclusions of the VR. However, it is not systematically implemented. The mobilization of this visit should be optimized.

Key words: occupational health, prereinstatement visit, preservation of employment

Contexte : La question du maintien dans l'emploi est au cœur des missions des équipes de santé au travail. La visite de préreprise (VPR) fait partie des démarches à déclencher précocement dans cet objectif. L'objectif principal de ce travail est d'étudier les modalités réelles de mise en œuvre de la VPR.

Méthode : Etude prospective, descriptive, en deux temps. $1^{\mathrm{er}}$ temps : questionnaire rempli lors de la VPR, par des médecins du travail d'un service de santé au travail interentreprises, pendant un mois. $2^{\text {ème }}$ temps : 6 mois après cette VPR, étude du devenir de ces salariés par l'analyse de données informatiques des conclusions des visites de reprise (VR) le cas échéant, et par téléphone.

Résultats : 172 questionnaires de VPR analysés (48\% des femmes ; l'âge moyen - 43,7 ans ; $56 \%$ des employés) : $67 \%$ des salariés vus en VPR pour la $1^{\text {ère }}$ fois, $51 \%$ en arrêt de travail depuis plus de 6 mois; $58 \%$ des VPR à l'initiative des salariés. Lors de la VPR, un aménagement des conditions de travail était préconisé dans $21 \%$ des cas et du temps de travail dans 19\% des cas. Sur les 116 salariés vus en VR, 50\% bénéficiaient d'un aménagement du poste de travail, 34\% étaient déclarés inaptes. La conclusion de la VR était significativement différente en fonction de l'existence de démarches préconisées par le médecin du travail lors de la VPR ( $p=0,034)$, d'un contact pris avec les intervenants externes à l'entreprise $(p=0,03)$, ou avec l'employeur $(p=0,017)$, et du type de pathologie $(p=0,04)$. Le devenir du salarié recueilli par téléphone était significativement différent en fonction du sexe et de l'existence d'un contact avec l'employeur ( $\mathrm{p}=0,017$ ), les autres croisements étant non significatifs.

Conclusion : La VPR est une étape clé pour le retour et le maintien au travail et conditionne les conclusions de la VR. Toutefois, elle n'est pas systématiquement mise en œuvre. Sa mobilisation devrait être optimisée.

Mots clés : santé au travail, visite de préreprise, maintien dans l'emploi 


\section{Introduction}

Dans le langage courant, le maintien dans l'emploi, notion assez récente, est considéré comme la prévention de la désinsertion professionnelle, c'est à dire la prévention du risque de perte d'emploi d'un salarié à la suite d'une altération de sa santé. Cela représente une inquiétude importante pour les pays, notamment en Europe et en France, et constitue un enjeu majeur de santé globale, avec des axes d'actions à mettre en place et à améliorer, ce dont attestent les priorités dégagées dans Plan National Santé Travail 3(1).

En Allemagne, la politique de maintien dans l'emploi est définie par un protocole national encadré par la loi, avec le BEM (Betriebliches Eingliederungs Management), qui est un dispositif de gestion concertée de maintien dans l'emploi obligeant les entreprises à réintégrer les salariés en situation de maladie prolongée, pour tous les salariés de toutes les entreprises privées et publiques. Dès 42 jours d'arrêt maladie dans l'année, d'origine professionnelle et non professionnelle, (consécutifs ou non), l'employeur doit prendre des mesures pour faciliter le retour dans l'emploi, et contacter le salarié (2).

Au Danemark, le maintien dans l'emploi et la réadaptation professionnelle relèvent de la compétence des communes qui sont chargées du suivi des arrêts de travail depuis la loi de 2007. Un 1er suivi est effectué par questionnaire. Un entretien a lieu dans tous les cas dans un délai maximum de 8 semaines. La commune prend alors contact avec l'entreprise pour envisager les perspectives de retour au travail. Parallèlement, depuis la loi sur l'absentéisme de 2009, l'employeur doit s'entretenir avec le salarié au plus tard la 4ème semaine d'arrêt de travail afin de connaître la durée provisoire de l'arrêt de travail, les capacités de travail, la possibilité d'aménagements à prévoir, et rapporter certaines informations au service de la commune (2).

En 2008, en Suède, une réforme visant à lutter contre l'attribution «d'aides passives» et à mettre en œuvre «des aides actives» pour prévenir la désinsertion professionnelle permettait une régression de $40 \%$ du taux d'absence pour maladie, et de $25 \%$ du nombre de nouveaux bénéficiaires de l'assurance invalidité (2).

La problématique du maintien dans l'emploi est également une préoccupation majeure en France. Plusieurs outils et acteurs œuvrent dans cet objectif.

Parmi ces acteurs, les services de santé au travail, dont une des missions principales est le maintien dans l'emploi, jouent un rôle majeur et doivent intervenir le plus en amont possible pour agir de manière efficace. C'est dans cette perspective qu'en cas d'arrêt de travail, la visite de préreprise (VPR) prend tout son sens : «En vue de favoriser le maintien dans l'emploi des salariés en arrêt de travail d'une durée de plus de trois mois, une visite de préreprise est organisée par le médecin du travail à l'initiative du médecin traitant, du médecin conseil des organismes de sécurité sociale ou du salarié» (article R4624-20 du CT).

«Au cours de l'examen de préreprise, le médecin du travail peut recommander:

$1^{\circ}$ Des aménagements et adaptations du poste de travail ;

$2^{\circ}$ Des préconisations de reclassement ;

$3^{\circ}$ Des formations professionnelles à organiser en vue de faciliter le reclassement du salarié ou sa réorientation professionnelle.

A cet effet, il s'appuie sur le service social du travail du service de santé au travail interentreprises ou sur celui de l'entreprise.

Sauf opposition du salarié, il informe l'employeur et le médecin conseil de ces recommandations afin que toutes les mesures soient mises en œuvre en vue de favoriser le maintien dans l'emploi du salarié» (article R4624-21 du CT).

De plus, selon l'article R4624-23, «L'examen de reprise a pour objet : 
$1^{\circ}$ De délivrer l'avis d'aptitude médicale du salarié à reprendre son poste ;

$2^{\circ}$ De préconiser l'aménagement, l'adaptation du poste ou le reclassement du salarié;

$3^{\circ}$ D'examiner les propositions d'aménagement, d'adaptation du poste ou de reclassement faites par l'employeur à la suite des préconisations émises par le médecin du travail lors de la visite de préreprise.[...]».

Cet article souligne qu'à la suite de la VPR l'employeur ne peut rester inactif et doit faire des propositions, qui seront ensuite examinées par le médecin du travail, ce qui renforce l'impact de cette visite (3).

Selon le bilan des conditions de travail 2013, établi par le Conseil d'orientation sur les conditions de travail (COCT), dans la répartition des visites médicales effectuées en 2012 (données issues des RAM sur 11 régions), les VPR représentaient 2,6\% des visites, soit 147 218 VPR. Et, selon une publication de 2013 de la DARES, en 2011, le taux d'absentéisme dans les entreprises françaises, tous salariés et tous secteurs confondus, était de 3,6\%, soit 14 jours d'absence par salarié dans l'année (pour cause de maladie, accident, ou garde d'enfants malade).

Les VPR actuellement représentent donc, sans doute, une toute petite part du nombre de VPR qui pourraient être réalisées.

En 2014, Fassier et coll. ont publié une étude concluant que la visite de préreprise est une des mesures de 1ère intention pour aider un salarié à garder son emploi malgré ses douleurs (4).

En 2013, Saliba et coll., lors d'entretiens semi-directifs avec des professionnels de santé, avaient déjà signalé l'importance de l'anticipation de la visite de préreprise (5).

Fau-Prudhomot et coll. ont réalisé une enquête sur le rôle du service de santé au travail dans le retour et le maintien dans l'emploi de salariés ayant eu une affection cancéreuse. Un tiers des salariés avaient bénéficié d'une visite de préreprise, et ils mettaient en évidence l'importance de cette visite dans le retour à l'emploi (6).

En 2014, Brasseur remarquait « une assez faible utilisation des visites de préreprise auprès des médecins du travail, [...], sans parler de la coordination entre le médecin traitant et le service de santé au travail inexistante dans une immense majorité de situations » (7).

En 2015, Sevellec et coll. concluaient quant à eux, que seuls $22 \%$ des salariées questionnées dans une enquête sur la reprise du travail après un cancer du sein, avaient bénéficié d'une visite de préreprise (8). La même année, Rollin et coll. étudiaient l'intérêt d'une consultation spécialisée d'aide à la reprise du travail après cancer. La visite de préreprise était conseillée dans $45 \%$ des consultations, mais réalisée uniquement dans $49 \%$ des cas avec un taux de réalisation inférieur aux autres conseils (donnée non significative) (9).

Nous avons donc souhaité mieux comprendre l'impact de la VPR afin d'inciter à une plus grande généralisation de son utilisation.

L'objectif principal de ce travail est d'étudier les modalités réelles de mise en œuvre de la VPR. Les objectifs secondaires sont d'évaluer l'impact de la VPR pour éviter la désinsertion professionnelle, d'identifier les difficultés liées à son déroulement et aux démarches préconisées, et d'optimiser les recommandations pour son application.

\section{Matériels et méthodes}

\section{Déroulement}

Après obtention de l'accord de la direction d'un Service de Santé au Travail de 150 médecins du travail (Pôle Santé Travail) du Nord de la France, un questionnaire a d'abord été présenté 
aux médecins du travail (et ajusté en fonction de leurs remarques), puis envoyé par mail en expliquant l'intérêt de l'étude, ainsi qu'à toutes les secrétaires et référents de secteur en joignant le questionnaire. Il était proposé aux médecins du travail volontaires de remplir un questionnaire (anonyme) lors de chaque VPR (une partie administrative à remplir par la secrétaire médicale et une partie à remplir par le médecin du travail). La période de cette première phase (=T0) de l'étude a été fixée du 12 novembre 2014 au 12 décembre 2014.

Lors d'une 2ème phase, à 6 mois des VPR (= T1), grâce au numéro de dossier reporté sur le questionnaire, il était prévu un recueil de données sur le logiciel médical du service de santé au travail (Stetho) afin de déterminer si les salariés avaient été revus dans ce délai en visite de reprise, et le cas échéant les conclusions de cette visite de reprise, ou s'ils avaient été revus de nouveau en VPR.

Puis, afin d'affiner les éléments recueillis, et d'évaluer les démarches préconisées lors de la VPR et réellement mises en œuvre, il a été décidé de contacter les salariés par appel téléphonique pour qui un questionnaire avait été rempli en VPR. Un mail a donc été envoyé aux médecins du travail ayant participé à l'étude pour les avertir du contact qui serait pris avec leurs salariés afin que ces derniers puissent exprimer leur avis, désaccord voire leur refus.

\section{Création des questionnaires}

Le questionnaire T0 d'une page est divisé en 2 parties distinctes. Une 1ère partie était à remplir par la secrétaire médicale lors de l'accueil du salarié regroupant des informations générales : données sociodémographiques, informations concernant l'entreprise, l'arrêt de travail, et le nombre de VPR. Ces données ont été évaluées par différentes variables. A des fins de comparaison, la classification de certaines de ces variables a été calquée sur celle de l'enquête du réseau régional Santé Travail Maintien dans l'Emploi (STME) Nord-Pas-deCalais (10).

Une 2ème partie était à remplir par le médecin du travail lors de la VPR. Cette partie renseigne le type de pathologie motivant l'arrêt de travail, la personne à l'initiative de l'arrêt de travail, les conclusions de la VPR et les difficultés liées à cette dernière.

Le questionnaire T1 d'une page est divisé en 2 parties. Une 1ère partie d'analyse de dossiers sur le logiciel Stetho, permettait de quantifier les variables «visite de reprise» et «VPR» dans l'intervalle, éventuellement les conclusions de la visite de reprise et dans ce cas le maintien des préconisations du médecin du travail (lorsque la conclusion de la visite de reprise correspondait totalement ou en parti aux préconisations du médecin du travail reportées sur le questionnaire T0), et la sortie éventuelle du salarié de l'entreprise.

Dans la 2ème partie, lors du contact téléphonique des salariés, leur situation professionnelle était analysée par les variables «arrêt de travail », «reprise du travail », "sorti de l'entreprise » ou «attente de licenciement ». Puis les autres démarches préconisées lors de la VPR effectuées étaient répertoriées.

\section{Facteurs d'inclusions, d'exclusions et perdus de vue}

Etaient inclus les questionnaires pour lesquels une VPR avait été réalisée et qui avaient été transmis par courrier interne ou par mail.

A T0, étaient exclus les questionnaires pour lesquels une autre visite que la VPR était réalisée, et les questionnaires avec plus de $50 \%$ de données manquantes.

A T1-analyse des données du logiciel Stetho, étaient considérés comme perdus de vue les questionnaires n'ayant pas de numéro de dossier Stetho renseigné ou un mauvais numéro 
Stetho, ne permettant pas de récupérer les données sur le logiciel médical. A T1-contact téléphonique, étaient considérés comme perdus de vue les questionnaires pour lesquels aucun numéro de téléphone n'était renseigné sur le logiciel ou sur les pages blanches ou ceux qui comportaient un mauvais numéro. Etaient perdus de vue également les salariés n'ayant pas répondu au téléphone après 2 rappels et ceux qui n'ont pas voulu répondre aux questions. Etaient exclus les salariés pour qui les médecins du travail n'ont pas souhaité que les salariés soient appelés.

\section{Analyses statistiques}

Il s'agit d'une étude prospective, observationnelle, descriptive, longitudinale (Niveau 4 de preuve scientifique). Le logiciel utilisé a été «Epi info ». L'analyse a comporté 2 étapes :

- Dans la lère étape, il s'agit de statistiques descriptives de la population étudiée.

- La 2ème étape correspondait à la comparabilité de différentes variables qualitatives (analyse bivariée) à l'aide d'un test du Khi-deux, avec calcul de la significativité statistique (p). L'analyse concernait les liens éventuels entre les conclusions de la VR, d'une part, et différents éléments recueillis lors de la VPR, d'autre part: le sexe du salarié, les différentes catégories d'âge, le niveau de formation du salarié, le poste de travail, l'ancienneté au poste et dans l'entreprise, l'effectif de l'entreprise, l'origine de l'arrêt de travail, la durée de l'arrêt de travail, le type de pathologie, l'existence d'un contact entre le médecin du travail et l'employeur ou les intervenants externes à l'entreprise, la préconisation de démarches par le médecin du travail lors de la VPR.

Eléments de confidentialité : Une information a été passée au salarié par chaque médecin lors de la passation du questionnaire concernant l'étude et la confidentialité des données (nous n'avons retenu dans les données administratives que le numéro de dossier du salarié nécessaire à la $2^{\text {ème }}$ étape de l'étude, pour le recueil de données sur le dossier informatique).

\section{Résultats}

175 questionnaires ont été récupérés et 172 étaient exploitables pour la $1^{\text {ère }}$ partie (T0); pour la $2^{\text {ème }}$ partie (T1), 161 ont été analysés sur logiciel médical informatique, et 90 salariés ont été contactés par téléphone.

\section{Population}

La moyenne d'âge de la population incluse est de 43,7 ans (minimum 20 ans et maximum 63 ans). La catégorie d'âge des salariés la plus représentée est celle comprise entre 45 et 54 ans $(\mathrm{N}=65 ; 38 \%)$, et la population comprend presque autant de femmes ( $\mathrm{N}=82 ; 48 \%)$ que d'hommes $(\mathrm{N}=90 ; 52 \%)$.

Les salariés inclus sont employés par des entreprises issues principalement du secteur d'activité du commerce de gros et de détails $(\mathrm{N}=32 ; 19 \%)$, de la construction $(\mathrm{N}=28 ; 16 \%)$ et des activités pour la santé humaine et l'action sociale sans hébergement $(\mathrm{N}=19 ; 11 \%$ pour les 2). La taille des entreprises concernées par ces salariés inclus est, pour $91 \%$ d'entre elles, supérieure à 10 salariés (un tiers entre 10 et 49 salariés, un tiers entre 50 et 199 et un tiers de plus de 200 salariés).

La majorité des salariés sont des employés $(\mathrm{N}=96 ; 56 \%)$ et des ouvriers ( $\mathrm{N}=46 ; 27 \%)$, et sont répartis de manière relativement homogène sur les 3 catégories d'ancienneté au poste de travail et dans l'entreprise (moins de 5 ans, entre 5 et 10 ans et plus de 10 ans d'ancienneté). La moyenne d'ancienneté est de 9,38 ans au poste de travail et de 10,87 ans dans l'entreprise 
(minimum 1 an et maximum 42 ans d'ancienneté pour les 2).

Plus de la moitié des salariés ont un niveau de formation CAP, BEP ou Baccalauréat ( $\mathrm{N}=93$; $54 \%)$.

Dans $67 \%$ des cas, il s'agit de la 1ère VPR $(\mathrm{N}=118)$, dans $19 \%$ des cas de la 2ème VPR $(\mathrm{N}=33)$, et dans $12 \%$ des cas, c'est une $3^{\text {ème }}, 4^{\text {ème }}$ ou $5^{\text {ème }}$ VPR $(\mathrm{N}=18)$, (données manquantes $\mathrm{N}=3$ ). Plus de la moitié des travailleurs sont en arrêt de travail depuis plus de 6 mois au début de l'étude, donc lors de la VPR $(\mathrm{N}=88 ; 51 \%)$; pour un tiers des salariés, la durée de l'arrêt de travail varie entre 1 et 3 mois $(\mathrm{N}=51 ; 30 \%)$.

Concernant les salariés pour qui il est réalisé une 1ère VPR $(\mathrm{N}=118)$, la durée des arrêts de travail varie de 3 à 1621 jours (moyenne $=234$ jours, médiane $=373$ jours), et la répartition entre les catégories de durées d'arrêt est sensiblement la même que pour tous les salariés réunis.

Dans $3 / 4$ des cas, les salariés sont en arrêt de travail pour maladie ordinaire $(\mathrm{N}=125 ; 73 \%)$, sinon, en arrêt de travail pour accident du travail et maladies professionnelle.

\section{La VPR}

Les pathologies occasionnant les arrêts de travail sont réparties en 3 grandes catégories (il faut noter qu'un même patient pouvait être en arrêt de travail pour plusieurs pathologies) (figure 1)

- TMS (rachis, membres supérieurs et membres inférieurs) $(\mathrm{N}=102 ; 50 \%)$,

- Maladies d'origine psychiatrique $(\mathrm{N}=57 ; 28 \%)$,

- Les autres pathologies $(\mathrm{N}=42 ; 22 \%)$.

Figure 1 : Pathologies à l'origine de l'arrêt de travail

Dans $15 \%$ des cas, 2 pathologies $(\mathrm{N}=27)$, et dans $1 \%$ des cas 3 pathologies différentes étaient rapportées $(\mathrm{N}=2)$.

Le salarié est à l'initiative de la VPR dans la majorité des cas $(\mathrm{N}=108$ soit $64 \%$, dont 9 sur conseil de l'employeur). Lorsque le médecin traitant est à l'origine de la VPR ( $\mathrm{N}=27 ; 16 \%)$, il a orienté son patient vers le médecin du travail avec un courrier dans $3 / 4$ des cas $(\mathrm{N}=20$; $75 \%)$, alors que l'initiative de la VPR par le médecin conseil $(\mathrm{N}=35 ; 20 \%)$ n'est accompagnée d'un courrier que dans la moitié des cas $(\mathrm{N}=17 ; 50 \%)$.

Dans la moitié des cas de VPR, la reprise du travail est déjà fixée ( $\mathrm{N}=86 ; 50 \%)$. Pour 30 salariés parmi ces 86, l'arrêt des indemnités journalières est prévu au moment de la VPR.

A l'issu de la VPR, le médecin du travail conclu que la reprise du travail ne peut avoir lieu immédiatement dans plus de la moitié des cas $(\mathrm{N}=101 ; 59 \%)$, le plus souvent dans l'attente/la poursuite de soins $(\mathrm{N}=51 ; 44 \%)$. Concernant les 86 salariés dont la reprise est déjà programmée, le médecin du travail se prononce dans 15 cas, à l'issue de la VPR, en faveur d'un report ultérieur de la reprise du travail.

Les autres éléments recensés justifiant la poursuite de l'arrêt de travail sont : l'attente d'avis de spécialistes médicaux, la demande de bilans de compétences et de formations; l'attente d'avis techniques qui comprennent un suivi psychologique, une étude ergonomique, l'avis d'un juriste, un bilan de pré-orientation, l'attente de fiches de données de sécurité.

Concernant les recommandations à l'issue de la VPR, les préconisations principales du médecin du travail sont des aménagements des conditions de travail $(\mathrm{N}=47 ; 21 \%$ dont 5 cas 
avec la mobilisation d'un service d'aide au maintien dans l'emploi) et du temps de travail $(\mathrm{N}=42 ; 19 \%)$.

Les autres préconisations du médecin du travail à l'issu de la VPR sont une étude de poste $(\mathrm{N}=16 ; 7 \%)$, une invalidité $(\mathrm{N}=10 ; 5 \%)$, un bilan de compétence ou une formation $(\mathrm{N}=30$; $14 \%)$, ou une RQTH $(\mathrm{N}=27 ; 12 \%)$, sachant que ces différentes démarches peuvent se cumuler pour un même salarié. Il faut noter que dans $28 \%$ des situations, aucune recommandation n'est effectuée à l'issue de la VPR.

Dans $61 \%$ des cas, une communication a lieu entre le médecin du travail et les différents contacts externes à l'entreprise : avec le médecin traitant du salarié ( $\mathrm{N}=43 ; 21 \%)$, le médecin conseil $(\mathrm{N}=21 ; 10 \%)$, le médecin spécialiste $(\mathrm{N}=22 ; 11 \%)$, l'assistante sociale $(\mathrm{N}=17 ; 8 \%)$; les autres contacts externes à l'entreprise correspondent à la référente handicap du SST, la cellule PDP (Prévention de la désinsertion professionnelle), ou le service formation. Un contact avec l'employeur est également établi dans $54 \%$ des cas $(\mathrm{N}=93)$, le plus souvent par courrier ou par mail $(\mathrm{N}=39 ; 23 \%)$.

Parmi les 86 salariés pour qui une visite de reprise du travail était programmée, 55 ont répondu à la question concernant les difficultés ressenties liées à la reprise du travail. Parmi les répondants, les salariés se sentent en capacité de reprendre le travail dans la grande majorité des cas $(\mathrm{N}=49 / 55 ; 89 \%)$.

Le médecin du travail perçoit le déroulement général de la VPR difficile dans 28\% (47/172) des cas, difficulté liée dans $28 \%$ des cas, soit 13/47 salariés, à la temporalité de la VPR par rapport à une date de reprise programmée, considérée comme trop proche.

Les difficultés liées au salarié sont dues, selon le médecin du travail, soit à un déni du salarié face à la situation, qui ne souhaite pas reprendre, ou au contraire qui pense pouvoir rester à son poste de travail malgré des capacités restantes restreintes, soit à la pathologie, soit à une souffrance psychologique et une forte appréhension en rapport avec la reprise du travail.

Concernant les difficultés liées à l'employeur, elles sont liées soit à une situation conflictuelle avec le salarié, soit à des difficultés de communication entre l'employeur et le médecin du travail, soit au fait que l'entreprise fait partie de la fonction publique, ce qui rend parfois plus difficile la communication avec l'employeur, ou la mise en place de certains aménagements (par exemple, pour les fonctionnaires, le TPT ne peut être accordé qu'après un arrêt de travail d'au moins 6 mois consécutifs de congés ordinaires de maladie pour une même affection ou pour congé longue maladie ou longue durée).

\section{Les suites de la VPR par l'analyse de dossiers médicaux informatiques}

A 6 mois de la VPR, nous avons réalisé une analyse des données sur le logiciel médical informatique pour recueillir les données sur la réalisation de nouvelles visites.

Sur les 161 dossiers de salariés analysés, 116 (72\%) ont bénéficié d'une visite de reprise. Parmi eux, 26 salariés (16\%) ont bénéficié d'au moins une nouvelle VPR entre la VPR initiale et la VR (6 salariés ont été revus 2 fois en VPR et 3 salariés, 3 fois). Parmi les 45 salariés (28\%) n'ayant pas bénéficié de visite de reprise, 18 (11\%) ont pu être revus lors d'au moins une nouvelle VPR ( 3 salariés ont été revus 2 fois et un salarié 3 fois).

Parmi les 116 salariés ayant bénéficié d'une visite de reprise du travail, dans la moitié des cas $(\mathrm{N}=58)$ la conclusion de la VR indiquée sur le dossier informatisé est une aptitude avec aménagement du poste de travail, et dans $34 \%$ une inaptitude $(\mathrm{N}=40)$ (Figure 2).

Figure 2: Conclusion de la visite de reprise 
Concernant les aménagements du poste de travail, les restrictions de tâches de travail et de postures concernent essentiellement la manutention $(\mathrm{N}=28 / 58 ; 47 \%)$, les postures contraignantes $(\mathrm{N}=12 / 58 ; 20 \%)$, et les mouvements répétitifs $(\mathrm{N}=5 / 58,8 \%)$. On retrouve également d'autres préconisations $(\mathrm{N}=14)$ : les efforts physiques intenses, les déplacements importants, la montée/descente d'escaliers, le travail en hauteur, le travail isolé, et la nécessité d'un poste administratif.

Les aides matérielles concernent la nécessité d'un véhicule avec boite automatique, d'un chariot de ménage, d'un siège assis debout, et d'un siège ergonomique.

Pour les dossiers où des préconisations ont été formulées lors de la VPR, les médecins du travail ont maintenu ces préconisations lors de la visite de reprise dans $72 \%$ des cas, et il y a donc une évolution de la décision préconisée lors de la VPR dans 28\% des cas.

Enfin, selon la base de données du logiciel Stetho, parmi les 161 dossiers analysés, $28 \%$ des salariés $(\mathrm{N}=45)$ apparaissent sortis de l'entreprise. Ce chiffre peut être sous-estimé car il dépend de la déclaration de l'employeur (cela signifie une fin de contrat, soit en raison d'un licenciement, soit de la fin d'un contrat à durée déterminée, soit une mise à la retraite, mais cette précision n'a pas pu être obtenue par le biais du logiciel informatique).

Par ailleurs, parmi les dossiers analysés sur le logiciel, pour les salariés ayant eu une visite de reprise $(\mathrm{N}=116), 5$ apparaissaient comme ayant bénéficié d'un nouvel arrêt de travail (car ils ont été revus de nouveau en VPR, ou en visite de reprise du travail); le reclassement apparaît mentionné seulement pour 2 salariés parmi les 40 déclarés inaptes.

Néanmoins, comme le logiciel Stétho ne comprend pas toutes les informations, nous avons décidé de compléter l'enquête par un contact téléphonique avec le salarié pour affiner ces données.

\section{Contact téléphonique des salariés}

Suite au recueil de données sur le logiciel, 90 salariés sur les 146 salariés contactés par téléphone (62\%) ont répondu à l'enquête téléphonique.

Cette sous-population était comparable à la population globale pour les variables analysées sauf pour l'ancienneté, la conclusion de la VPR, et la pathologie à l'origine de cet arrêt (notamment pour les TMS pris globalement, mais les 3 sous-catégories de TMS rachis, membres supérieurs, et membres inférieurs étaient comparables).

$\mathrm{Au}$ total, parmi les répondants, un tiers ont déclaré être sortis de l'entreprise $(\mathrm{N}=30 ; 33 \%)$, dont la majorité par un licenciement pour impossibilité de reclassement dans le cadre d'une inaptitude médicale $(\mathrm{N}=23 ; 76 \%)$ (Tableau 1), le reste $(24 \%)$ suite à une rupture conventionnelle $(\mathrm{N}=3 ; 10 \%)$, une démission $(\mathrm{N}=2 ; 7 \%)$ ou une fin de contrat $(\mathrm{N}=2 ; 7 \%)$. Le ratio hommes/femmes pour les salariés sortis de l'entreprise est 1 femme pour 2 hommes. Parmi les salariés restés dans l'entreprise $(\mathrm{N}=60,67 \%), 22$ sont encore en arrêt de travail $(37 \%)$ et $38(63 \%)$ ont repris le travail.

Pour les salariés en arrêt de travail $(\mathrm{N}=22), 23 \%$ souffrent d'une pathologie différente que celle ayant occasionné l'arrêt de travail lors de la VPR (N=5), 95\% sont toujours en cours de soins $(\mathrm{N}=21), 95 \%$ ont bénéficié d'un avis par un médecin spécialiste $(\mathrm{N}=21)$, et $77 \%$ ont rencontré le médecin conseil $(\mathrm{N}=17)$.

Concernant les salariés ayant repris le travail $(\mathrm{N}=38), 8 \%$ ont bénéficié de l'intervention du SAMETH $(\mathrm{N}=3), 24 \%$ ont été arrêtés de nouveau par leur médecin traitant dans l'intervalle, depuis la reprise du travail $(\mathrm{N}=9)$, avec quelques arrêts courts mais surtout des arrêts longs de plusieurs mois; et pour les salariés concernés $(\mathrm{N}=30)$, les préconisations du médecin du travail étaient totalement respectées par l'employeur dans $58 \%$ des cas $(\mathrm{N}=22)$, et en partie dans $13 \%$ des cas $(\mathrm{N}=5)$. L'employeur ne respectait donc pas du tout les préconisations dans 
$8 \%$ des cas $(\mathrm{N}=3)$

Tableau 1 : Situation professionnelle des salariés contactés

Pour les salariés sortis de l'entreprise, seuls $13 \%$ ont retrouvé un travail et ne recherchent plus d'emploi $(\mathrm{N}=4)$. Il y a tout de même $27 \%$ des personnes ne cherchant pas d'emploi, notamment pour les personnes proches de la retraite, ou pour les personnes qui ont une invalidité. Les autres sont toujours en recherche d'un emploi $(\mathrm{N}=18)$.

Tous les salariés $(\mathrm{N}=90)$ ont également été interrogés sur les démarches effectuées à la suite de la VPR et de la visite de reprise. On constate que la majorité des actions ont été menées à la suite de la VPR.

La RQTH a été demandée dans 21 situations à l'issue de la VPR, et 4 situations à l'issue de la VR (pour ces salariés la RQTH a été conseillée lors de la VPR dans 6 cas). L'invalidité a été demandée dans 8 cas à l'issue de la VPR et dans 6 cas à l'issue de la VR (l'invalidité a été conseillée lors de la VPR pour 5 d'entre eux). Mais il faut noter que 8 salariés bénéficiaient déjà de la RQTH et 10 salariés de l'invalidité avant la réalisation de la VPR. Par ailleurs, un salarié sur 2 n'a eu aucun contact avec son employeur pendant toute la durée de son arrêt de travail.

\section{Analyse bivariée des données}

Le tableau 2 concerne les 116 salariés ayant bénéficié d'une VR parmi les 161 dossiers informatiques analysés. Les chiffres en rouges dans ce tableau correspondent à ceux repris dans le texte.

Parmi les 116 VR analysées, il n'y avait pas de lien statistiquement significatif entre la conclusion de la VR d'une part et le sexe, les différentes catégories d'âge, de niveau de formation du salarié, de poste de travail, d'ancienneté au poste et dans l'entreprise, d'effectif de l'entreprise, d'origine de l'arrêt de travail, et de durée de l'arrêt de travail d'autre part $(\mathrm{p}>0,05)$.

Nous avons observé un lien significatif entre le fait d'avoir des démarches préconisées par le médecin du travail lors de la VPR, et la conclusion de la VR ( $\mathrm{p}=0,034)$ (Tableau 2).

Les avis d'aptitude avec réserve à l'issue de la VR étaient plus fréquents lorsque des préconisations avaient été posées lors de la VPR (54\% vs 37\%). Il y avait presque autant de cas avec et sans démarches préconisées pour les salariés inaptes.

Nous avons observé un lien significatif statistiquement $(\mathrm{p}=0,03)$ entre la conclusion de la VR, et le fait d'avoir établi un contact lors de la VPR entre le médecin du travail et les intervenants externes à l'entreprise (Tableau 2). En effet, nous avons constaté une association entre les difficultés lors de la reprise du travail et la fréquence des contacts établis par le médecin du travail lors de la VPR. Plus il y a eu de contacts à l'issue de la VPR, plus la reprise du travail a été difficile.

Il existe également une différence significative $(\mathrm{p}=0,017)$, entre les cas où un contact avait été établi entre le médecin du travail et l'employeur, par rapport à ceux où il n'y avait pas eu de contact. En cas d'aptitude, il y avait peu de contacts avec l'entreprise $(n=12 / 17 ; 70,6 \%)$; alors qu'au contraire en cas d'aptitude avec un aménagement du poste de travail, il y avait plus d'échanges avec l'employeur ( $\mathrm{N}=39 / 55 ; 70,9 \%)$ (Tableau 2). 
Concernant le lien entre la conclusion de la VR et le type de pathologies à l'origine de l'arrêt de travail, nous avons observé une différence significative $(\mathrm{p}=0,04)$ entre les différents groupes étudiés (tableau 2).

Pour les salariés aptes avec aménagements, ils souffraient principalement de TMS (N=33/58 ; $56,9 \%)$.

Parmi les salariés déclarés inaptes, les TMS étaient également majoritaires ( $N=21 / 40$; $52,5 \%)$.

Et parmi les personnes souffrant de pathologies psychiatriques, la majorité étaient déclarés inaptes à leur poste de travail $(\mathrm{N}=16 / 38 ; 42,1 \%)$.

\section{Discussion}

Ce travail nous permet de dégager quelques pistes d'action et de conforter d'autres déjà évoquées dans des travaux antérieurs, pouvant améliorer la prévention de la désinsertion professionnelle. Certains paramètres comme l'âge, le niveau de formation et la durée de l'arrêt de travail devraient constituer des points de vigilance dans le déclenchement d'une visite de préreprise. La communication entre les praticiens (médecins du travail, médecins soignants, médecins conseils), d'une part et entre les médecins du travail et les différents interlocuteurs de l'entreprise, d'autre part pourraient également être des points d'amélioration pour le maintien dans l'emploi.

Notre travail comporte en même temps des limites. Tout d'abord, pour des raisons organisationnelles, nous nous sommes limités à la passation du questionnaire dans un seul SST interentreprises. Nous pouvons supposer que les démarches entreprises lors des VPR dans des SST autonomes sont différentes du fait de la plus grande proximité avec les salariés et avec les interlocuteurs internes à l'entreprise. Toutefois, l'activité du SST interentreprises concerné dans l'étude, le 2ème plus grand de France après l'Ile-de-France, couvre une partie très importante du territoire du Nord-Pas-de-Calais et concerne tous les secteurs d'activité. Nous avons comparé nos résultats à la seule étude retrouvée dans la littérature analysant la VPR, publiée en 2011 dans le limousin (Moreau et coll.) (11), qui reposait sur 1138 questionnaires recueillis sur une période de 12 mois, alors que notre étude reposait, elle, sur 172 questionnaires recueillis en 1 mois.

Certains questionnaires à T0 comportaient des données manquantes, ceci pouvant sans doute être expliqué par le fait qu'ils ont été remplis par de multiples médecins du travail et secrétaires médicales. Certaines de ces données manquantes ont pu être récupérées lors du remplissage du questionnaire $\mathrm{T} 1$, à partir des données des dossiers informatiques. Les questionnaires à T1 étaient en revanche complets, sans doute grâce au fait d'avoir une seule personne dédiée à cette tâche.

Pour la 2ème partie de la $2^{\text {ème }}$ phase (T1), 69 salariés ont été perdus de vue et 2 exclus, soit 71 questionnaires sur 161 restants (44\%). On peut s'interroger sur la représentativité de ces salariés non joignables par téléphone, qui étaient différents de la population contactée, notamment pour le type de pathologie à l'origine de l'arrêt de travail; en effet, ils souffraient plus de troubles psychiatriques (44\% vs $26 \%$ ). De ce fait, on peut évoquer la possibilité d'un biais de sélection.

L'âge moyen des salariés reçus en VPR était de 43,7 ans (38\% entre 45 et 54 ans), ce qui est supérieur à l'âge moyen des actifs de la région en 2007, qui était de 37,3 ans. Dans notre étude, $67,5 \%$ des salariés déclarés inaptes avaient plus de 45 ans $(\mathrm{N}=27)$. La population de l'enquête de Moreau et coll. était plus âgée (38,7\% des salariés avaient entre 50 et 59 ans) 
(11).

Le réseau régional Santé Travail Maintien dans l'Emploi (STME) a développé un suivi d'indicateurs collectifs sur les inaptitudes en Nord-Pas-de-Calais, afin de favoriser le maintien en emploi des salariés en situation d'inaptitude, par l'information et le conseil des équipes de santé au travail (10). Nous avons établi quelques parallèles entre notre population vue en VPR et cette population suivie dans le cadre d'une inaptitude médicale puisqu' un certain nombre de VPR débouchent sur une inaptitude (et ce, même s'il ne s'agit pas de la même population). Dans leur enquête, la tranche d'âge la plus touchée par l'inaptitude est celle des plus de 45 ans $(\mathrm{N}=2016 ; 63,5 \%)$.

Ceci renforce l'idée que l'âge relativement avancé de la population vue en VPR doit amener à une grande vigilance, car il serait corrélé à de moindres possibilités de retour au travail, ce que confirment les données de la littérature (12).

Concernant la taille des entreprises, celles de plus de 200 salariés étaient les plus fréquentes dans notre enquête, alors qu'elles sont sous-représentées dans l'étude Inaptitude du réseau STME. Comme les travailleurs des entreprises de plus de 200 salariés sont surreprésentés dans la population vue en VPR et sous - représentée dans l'enquête STME, on peut donc supposer que non seulement ils connaissent mieux le dispositif VPR mais qu'en plus, les possibilités de maintien en emploi sont accrues du fait de la taille de l'entreprise. Cela a d'ailleurs été démontré par une étude canadienne, en 2009, qui a également effectué une revue de la littérature sur les facteurs facilitant le retour au travail, qui a mis en évidence que les travailleurs issus d'entreprises de grandes tailles retournent plus facilement au travail du fait d'aménagements et de relocalisations plus faciles (13).

L'enquête STME met en évidence que la population ouvrière est nettement plus touchée par l'inaptitude (10). Hors, dans notre étude, ce sont les employés qui sont le plus vus en VPR. Dans la publication de Mairiaux et coll., un travail physiquement lourd, et l'exposition à des contraintes biomécaniques sont retrouvés comme facteurs entravant ou retardant le retour au travail (12). On peut s'interroger sur la sous-représentation des ouvriers dans notre étude, alors que vraisemblablement, ils sont le plus exposés au risque d'inaptitude. Peut-être est-ce dû à un défaut d'information.

Concernant le niveau de formation, 54\% des salariés vus en VPR dans notre enquête avaient un faible niveau de formation, type CAP. Ceci est également retrouvé dans l'enquête Inaptitude du réseau STME (40,7\%), faisant penser que cette population ayant une «faible qualification » est peut-être plus à risque de désinsertion professionnelle, imposant une vigilance plus accrue de la démarche de maintien dans l'emploi. En effet, l'étude française récente de Pélissier et coll., a identifié, chez les travailleurs absents au moins 3 semaines, le niveau de qualification inférieur au niveau d'enseignement secondaire comme un facteur influençant négativement le retour au travail $(\mathrm{p}<0,01)(14)$. D'autres études ont retrouvé le faible niveau de qualification comme facteur pronostic, négativement associé au retour au travail, notamment Hankins et coll. pour les salariés après accident du travail (15), Van Muijen et coll. pour les survivants du cancer (16), et Donker-Cools et coll. pour les salariés après lésion cérébrale acquise (17).

Concernant la durée de l'arrêt de travail, elle était de plus de 6 mois pour $51 \%$ des salariés vus en VPR de notre étude, et pour $78 \%$ des salariés au moment de la déclaration de l'inaptitude dans l'enquête STME. Cela conforte la nécessité d'anticiper davantage les actions de maintien dans l'emploi, afin que les salariés soient vus en VPR plus tôt pendant l'arrêt de travail, dans la mesure où il est reconnu que plus l'arrêt maladie se prolonge, plus le risque de désinsertion augmente. Selon l'enquête «European working conditions surveys », les chances de reprise 
du travail sont inversement proportionnelles à la durée d'absence : moins de $50 \%$ dans le cadre d'un arrêt de 3 à 6 mois, moins de $20 \%$ dans le cadre d'un arrêt de plus d'un an, et moins de $10 \%$ dans le cadre d'un arrêt de plus de 24 mois (18).

La législation impose une VPR pour les arrêts de plus de 3 mois, mais il faut rappeler que cette visite peut avoir lieu avant ; on constate que dans notre étude $36 \%$ des salariés vus en VPR étaient en arrêt de travail depuis moins de 3 mois $(24,3 \%$ des salariés de l'étude de Moreau et coll.). Cela suggère que nombreux sont ceux qui ont compris que la visite de préreprise peut être mobilisée à tout moment d'un arrêt de travail sans attendre nécessairement le seuil de 3 mois d'arrêt.

Les salariés vus en VPR souffraient dans $50 \%$ des cas de TMS, et dans $28 \%$ des cas de pathologies psychiatriques, ce qui est très proche des résultats obtenus par Moreau et coll. (respectivement $48 \%$ et $24 \%$ ), et dans l'enquête STME (51\% et $23 \%$ ). De plus, dans notre étude, nous avons observé une différence significative de conclusion de la VR en fonction du type de pathologie (un résultat statistique est considéré comme significatif lorsqu'il est peu probable que ce résultat est dû au simple hasard ; le seuil de significativité d'un test statistique est noté « $\mathrm{p}$ » et habituellement il est fixé à $5 \%$, c'est-à-dire qu'un résultat est statistiquement significatif quand $\mathrm{p}<0,05)$.

Par ailleurs, $26 \%$ des pathologies dans l'étude étaient d'origine professionnelle $(30 \%$ dans l'enquête inaptitude), et $66,7 \%$ des salariés ayant déclaré une maladie professionnelle étaient inaptes à leur poste de travail lors de la VR.

Ces chiffres soulèvent la question de la prévention primaire des risques professionnels, en privilégiant les actions collectives au sein des entreprises afin de réduire les contraintes au travail à l'origine de ces pathologies.

Le médecin traitant était à l'origine de la VPR dans $16 \%$ des cas. Une publication de l'INPES en 2012 qui interrogeait les médecins généralistes, qui sont souvent les premiers consultés pour des problèmes de santé sur leur fréquence de sollicitation des médecins du travail, concluait que $49 \%$ des médecins généralistes sollicitaient une VPR si leur patient était en arrêt de travail prolongé, et $15 \%$ des médecins généralistes déclaraient ne jamais le faire. Parmi les médecins généralistes ayant adressé des patients à leurs médecins du travail sur les 12 mois précédent l'enquête, la moyenne de patients adressés par médecin était de 7,8 patients sur 12 mois (19). Ces résultats démontrent la nécessité d'informer davantage les médecins généralistes de la possibilité de réalisation de cette visite, de son intérêt, et donc de l'importance de leur rôle dans l'orientation de ces patients le plus précocement possible.

D'ailleurs, on mettait en évidence que les orientations par le médecin traitant et le médecin conseil n'étaient accompagnées d'aucun courrier, respectivement dans $25 \%$ et $50 \%$ des cas. De même, ces praticiens étaient contactés par le médecin du travail à l'issu de la VPR dans $21 \%$ des cas pour le médecin traitant, $10 \%$ des cas pour le médecin conseil et $11 \%$ des cas pour les médecins spécialistes.

Dans l'enquête STME, pour les salariés inaptes ayant été vus au préalable en VPR, un contact avait été pris avec le médecin traitant dans $13,5 \%$ des cas, et avec un médecin spécialiste dans $13,5 \%$ des cas. Par ailleurs, il y avait une différence significative $(\mathrm{p}=0,003)$ de la conclusion de la VR en fonction de la prise de contact, ou non, externe à l'entreprise, (y compris le contact avec les confrères) lors de la VPR.

Afin de parvenir à une meilleure prise en charge, il conviendrait peut être d'améliorer la communication entre les praticiens dans le respect des règles déontologiques.

Ces contacts étaient d'autant plus fréquents qu'il existait in fine des difficultés lors de la reprise (restrictions voire inaptitude). Nous ne pouvons pas faire de lien entre l'existence de ces contacts et une amélioration du maintien dans l'emploi, en même temps l'absence de ces contacts pourraient rendre le maintien encore plus difficile. Un contact a été pris par le 
médecin du travail avec l'employeur lors de la VPR dans 54\% des cas dans notre enquête; et seulement dans 15\% des cas des salariés vus en VPR de l'enquête STME. On peut regretter que le contact avec l'employeur ne soit pas plus systématique (en dehors des cas où le salarié s'y oppose) dans la mesure où l'on demande à l'employeur à la fois de l'anticipation, et de l'investissement dans la démarche de reclassement. Cette notion est confortée par le fait que, dans notre enquête, la prise de contact par le médecin du travail avec l'employeur joue un rôle significatif dans la conclusion de la VR (ainsi, on observe dans notre enquête que dans les cas où un contact a eu lieu avec l'employeur, $57 \%$ des salariés bénéficiaient d'un aménagement de leur poste de travail, et $34 \%$ étaient inaptes).

Parmi les 116 salariés vus en VR à la suite de la VPR, 34\% étaient déclarés inaptes par le médecin du travail, dont la quasi-totalité étaient sortis de l'entreprise, c'est à dire, licenciés. Pour ces 40 salariés inaptes, on peut supposer que la VPR a eu au moins le mérite de permettre au médecin du travail d'informer le salarié sur la procédure d'inaptitude, de le préparer à un éventuel reclassement, ou à la possibilité d'un licenciement. La VPR a également permis de préconiser 5 demandes de mise en invalidité, 8 demandes de RQTH, et 14 demandes de bilans de compétences ou de formations, autant de démarches qui, si elles n'ont pu empêcher la perte d'emploi, pourront faciliter une réinsertion ou une reconversion professionnelle, ou au moins un relais pour obtenir des revenus de substitution. Il aurait été intéressant de savoir si les salariés inaptes après un arrêt maladie sont davantage reclassés lorsqu'ils ont bénéficié d'une VPR que sans.

Parmi les 90 salariés contactés par téléphone, une RQTH a été demandée pour 25 salariés lors de la VPR ou de la VR, et 8 salariés bénéficiaient déjà de ce statut avant la VPR, ce qui correspond à $37 \%$ de salariés appelés reconnus comme travailleurs handicapés. Dans l'étude de Moreau et coll., une RQTH était demandée auprès de la MDPH, à l'issue de la VPR, dans $24,6 \%$ des cas. De même l'invalidité ou l'intervention du SAMETH ont été peu sollicités. Si bien sûr on peut s'interroger sur la volonté du salarié de mobiliser systématiquement ces aides, en regard, la question de l'utilisation optimale de toutes les ressources disponibles afin d'assurer le maintien dans l'emploi des salariés se pose également. En ce sens, la Loi Rebsamen et l'aménagement de l'article L4624-1 du Code du travail peut constituer une évolution favorable dans la mesure où il préconise que toute préconisation du médecin du travail peut (c'est à dire chaque fois que nécessaire et possible) s'assortir de la mobilisation de l'équipe pluridisciplinaire en vue de la prévention de la désinsertion professionnelle.

\section{Conclusion}

Dans un but de prévention de la désinsertion professionnelle, la VPR s'inscrit comme un des principaux outils de la démarche de maintien dans l'emploi pour les SST.

Franche et coll., dans leur revue de la littérature sur les éléments impactant le retour au travail, ont mis en évidence, parmi les facteurs facilitants, le contact précoce avec le salarié en arrêt de travail et la présence d'un coordonnateur du retour au travail (20). La VPR pour un contact précoce, et l'équipe de santé au travail avec le médecin du travail comme coordonnateur du retour au travail devraient avoir une place primordiale en France pour favoriser le retour et le maintien en emploi.

Les préconisations du médecin du travail, en amont de la reprise, ont un impact sur la conclusion de la visite de reprise ; l'intérêt de la VPR est d'autant plus indéniable et doit être affirmé à chaque occasion.

Certains points restent tout de même à améliorer, notamment une plus grande systématisation 
des VPR lors des arrêts de travail pouvant engendrer des incapacités de travail même temporaires (y compris les arrêts de travail inférieurs à 3 mois), associée à une meilleure communication avec les médecins soignants, le recours à certains outils sous-utilisés, et des échanges plus fréquents avec l'entreprise dans l'objectif de construire ensemble une stratégie de retour au travail et de maintien dans l'emploi.

Un guide pratique utilisable à l'échelle nationale pourrait permettre une harmonisation des pratiques par exemple, en application du plan régional santé travail 3.

Une information systématique sur la VPR, à formaliser par un contact obligatoire de l'employeur à son salarié, pourrait permettre à tous les salariés en arrêt de travail d'être informés de la possibilité de réalisation de cette visite.

\section{Points essentiels}

La question du maintien dans l'emploi, enjeu majeur de santé globale et axe prioritaire d'action dans le Plan National Santé Travail 3, est au cœur des missions des équipes de santé au travail,

Etude prospective, deseriptive, par questionnaire, l'objectif principal de ce travail est d'étudier les modalités réelles de mise en @uvre de la VPR. Les objectifs secondaires sont d'évaluer l'impact de la VPR pour éviter la désinsertion professionnelle, en étudiant le devenir de ces salariés 6 mois après cette VPR, d'identifier les difficultés liées à son déroulement et aux démarches préconisées, et d'optimiser les recommandations pour son application.

Ce travail nous permet de dégager quelques pistes d'action et de conforter d'autres déjà évoquées dans des travaux antérieurs, pouvant améliorer la prévention de la désinsertion professionnelle. La visite de préreprise (VPR) est une étape clé à déclencher précocement dans cet objectif. Toutefois, elle n'est pas encore assez systématiquement mise en œuvre. Sa mobilisation devrait être optimisée, car elle conditionne les conclusions de la visite de reprise. @. Tout d'abord, une information systématique par le service de santé au travail ou l'employeur sur la VPR, à formaliser, pourrait permettre à tous les salariés en arrêt de travail d'être informés de la possibilité de réalisation de cette visite. Ensuite, certains paramètres comme l'âge (surtout au-delà de 45 ans), le faible niveau de qualification et la durée de l'arrêt de travail (y compris les arrêts de travail inférieurs à 3 mois) devraient constituer des points 
de vigilance dans le déclenchement d'une visite de préreprise. Enfin, une meilleure communication entre les praticiens (médecins du travail, médecins soignants, médecins conseils), d'une part et entre les médecins du travail et les différents interlocuteurs de l'entreprise, d'autre part pourraient également être des points d'amélioration pour le maintien dans l'emploi. 


\section{Références bibliographiques}

(1) Plan de santé au travail 2016-2020 (PST 3) - Plans de santé au travail Ministère du Travail, de l'Emploi, de la Formation professionnelle et du Dialogue social ». Consulté le 7 janvier 2016. http://travail-emploi.gouv.fr/sante-au-travail/plans-de-sante-autravail/article/plan-de-sante-au-travail-2016-2020-pst-3.

(2) EUROGIP. Prévention de la désinsertion professionnelle: l'expérience de 6 pays. EUROGIP- Note Thématique. 2010;(52/F):60.

(3) Czuba, C., et S. Fantoni-Quinton. «Maintien dans l'emploi après la réforme de la santé au travail : un effort renouvelé ». Archives des Maladies Professionnelles et de l'Environnement 74, nº 5 (novembre 2013): 515-18. doi:10.1016/j.admp.2013.07.186.

(4) Fassier J-B, Petit A, Yven C, Sappey P, Bernieri F, Soyeux E, et al. Douleurs et travail : aider le patient à garder un emploi. Douleurs Eval - Diagn - Trait. févr 2014;15(1):11-9.

(5) Saliba M-L, Arnaud S, Souville M, Viau A, François G, Verger P. Pratiques et perceptions des professionnels vis-à-vis du dispositif de maintien dans l'emploi : étude qualitative en région Provence-Alpes-Côte d'Azur. Rev DÉpidémiologie Santé Publique. avr 2013;61(2):172-9.

(6) Fau-Pruhomot P, Alcouffe J, Leroy C, Mora victoria, Sanchez-Bréchot M-L, Trimbach M, et al. Le rôle du service de santé au travail dans le retour et le maintien dans l'emploi de salariés ayant eu une affection cancéreuse. Enquête préliminaire. CAMIPInfo. 2012;(4).

(7) Brasseur, Bondeelle, Carlier, Plisson, Ravallec, Vaudoux. La réinsertion professionnelle. Trav Sécurité. sept 2014;(753):15-29.

(8) Sevellec M, Belin L, Le Bidault S, Stakowski H, Cotasson F, Bourrillon M-F, et al. Reprise du travail après cancer du sein. Arch Mal Prof Environ. févr 2015;76(1):47-8.

(9) Rollin L, De Blasi G, Boucher L, Gehanno J-F. Intérêts d'une consultation spécialisée d'aide à la reprise du travail après cancer. Bull Cancer (Paris). févr 2015;102(2):182-9.

(10) STME. Suivi d'indicateurs sur les inaptitudes en Nord-Pas-de-Calais. ISTNF; 2012.

(11) Moreau D. Étude régionale sur la visite de pré reprise, outil majeur dans la prévention contre la désinsertion professionnelle. Arch Mal Prof Environ. juin 2012;73(3):582.

(12) Mairiaux P, Schippers N, Kefer F, Cornelis S, Somville P, Donceel P. Retour au travail après une absence de longue durée [Internet]. SPF Emploi \& Travail; 2012.

(13) Kane S. Épuisement professionnel: facteurs facilitant et entravant le retour au travail. 2009.

(14) Pélissier C, Fontana L, Chauvin F. Factors influencing return to work after illness in France. Occup Med. 1 janv 2014;64(1):56-63.

(15) Hankins AB, Reid CA. Development and Validation of a Clinical Prediction Rule of the Return-to-Work Status of Injured Employees in Minnesota. J Occup Rehabil. sept 2015;25(3):599-616.

(16) Van Muijen P, Weevers NLEC, Snels I a. K, Duijts SFA, Bruinvels DJ, Schellart AJM, et al. Predictors of return to work and employment in cancer survivors: a systematic review. Eur J Cancer Care (Engl). mars 2013;22(2):144-60.

(17) Donker-Cools BHPM, Wind H, Frings-Dresen MHW. Prognostic factors of return to work after traumatic or non-traumatic acquired brain injury. Disabil Rehabil. 3 juill 2015;1-9.

(18) Eurofound. European Working Conditions Surveys. EWCS. 2005.

(19) Verger P, Ménard C, Richard J-B, Viau A. Médecins généralistes et santé au travail. INPES - Etudes Santé. (Janvier 2012):115-86.

(20) Franche R-L, Baril R, Shaw W, Nicholas M, Loisel P. Workplace-based return-to-work interventions: optimizing the role of stakeholders in implementation and research. J Occup Rehabil. 2005;15(4):525-42. 


\section{Annexe 1 : schéma morphologique de l'étude}

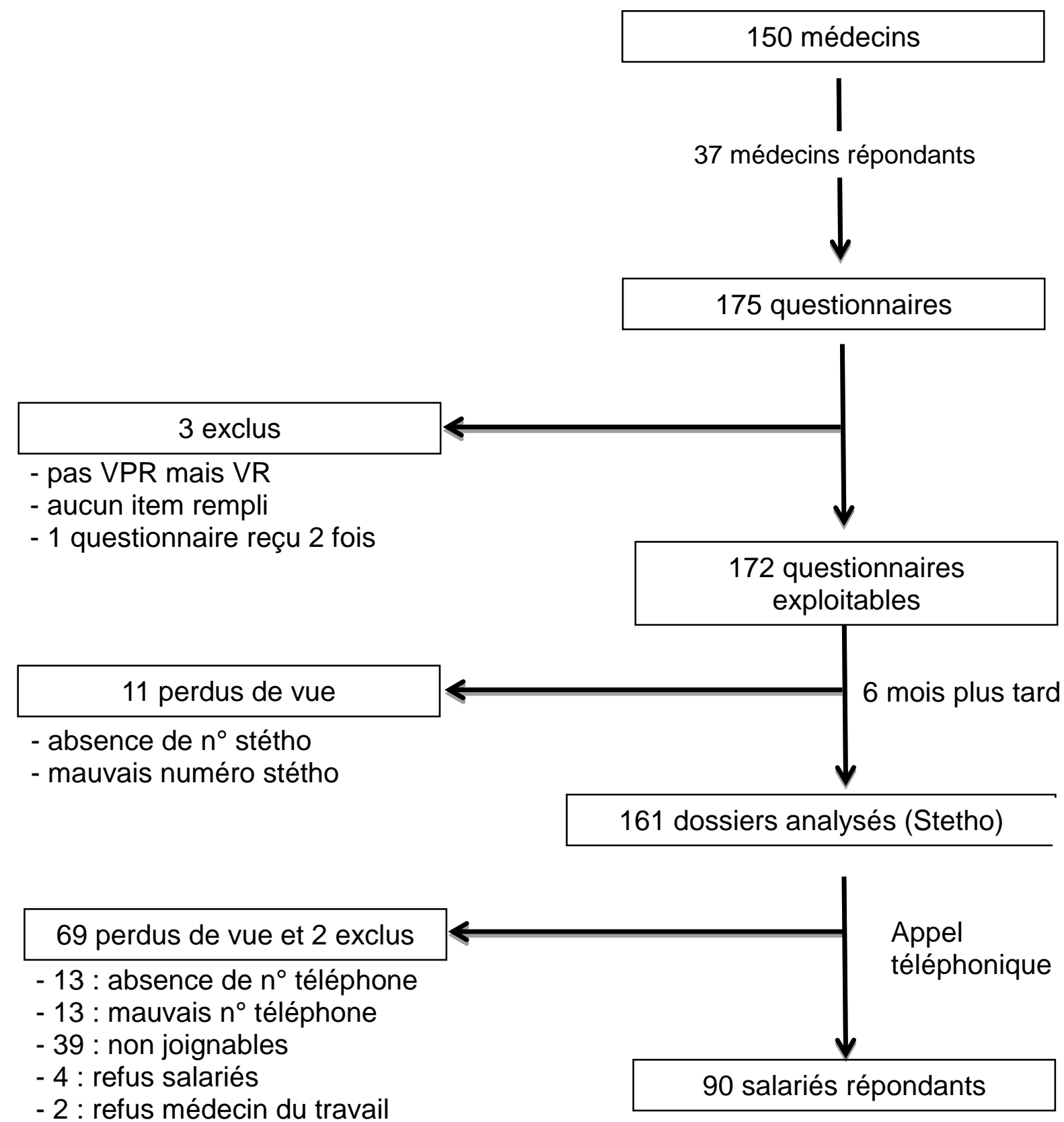


Figure 1 : Pathologies à l'origine de l'arrêt de travail

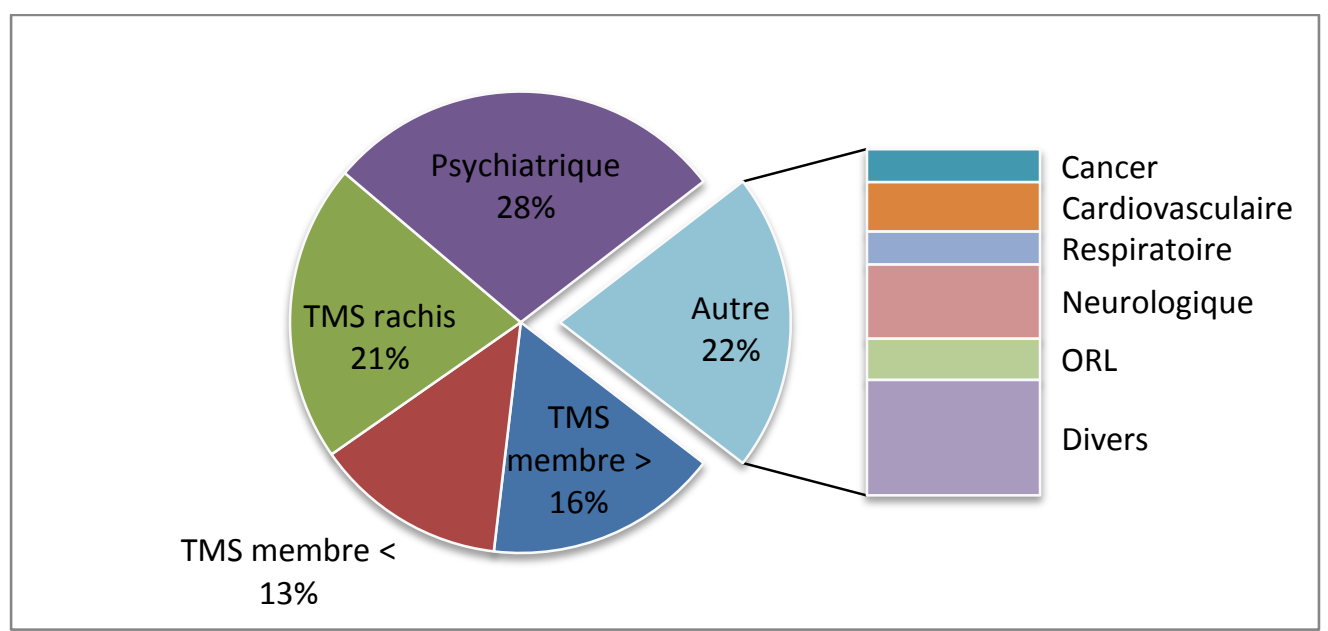

Figure 2 : Conclusion de la visite de reprise

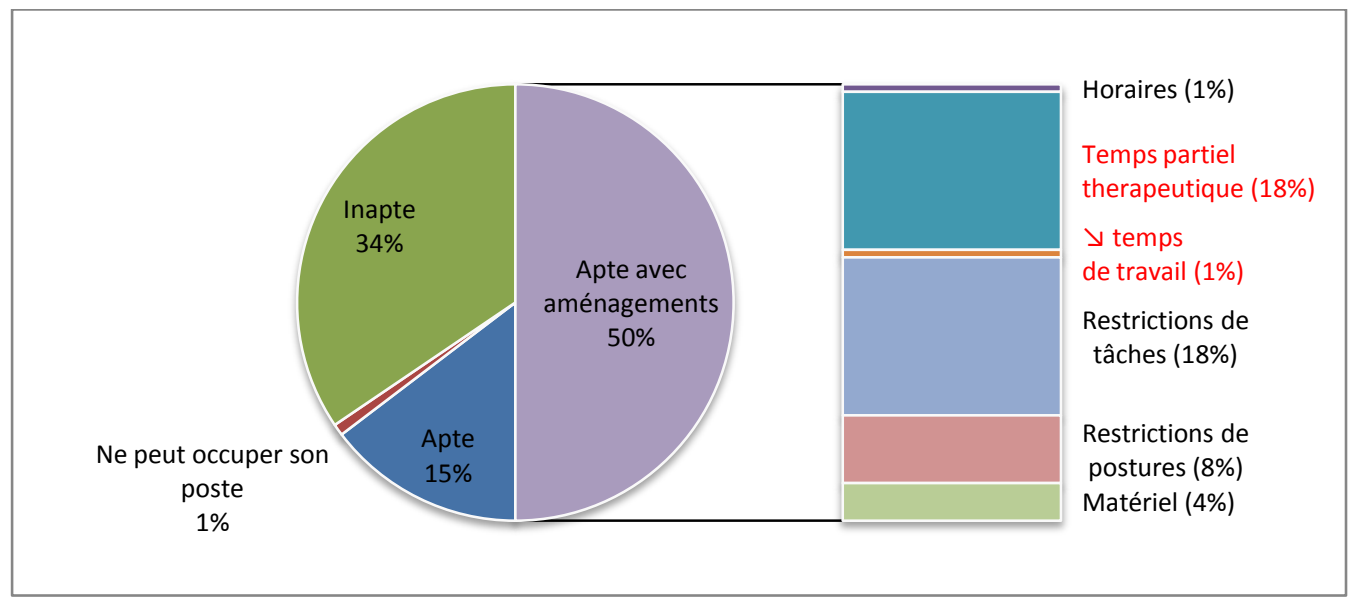

Tableau 1 : Situation professionnelle des salariés contactés

\begin{tabular}{|l|l|l|l|}
\hline Situation n,\{\%\} & Femmes & Hommes & Total \\
\hline En arrêt de travail & $9\{10\}$ & $13\{15\}$ & $22\{25\}$ \\
\hline Reprise du travail & $23\{25\}$ & $15\{17\}$ & $38\{42\}$ \\
\hline Sorti de l'entreprise & $10\{11\}$ & $20\{22\}$ & $30\{33\}$ \\
\hline Total & $42\{46\}$ & $48\{54\}$ & 90 \\
\hline
\end{tabular}

Tableau 2 : Croisements de la conclusion de la VR en fonction de certaines données de la 


\section{VPR}

tExemple de lecture du tableau (voir cadran) :

- «\% dans conclu»: 52,9\% des salariés déclarées aptes au même poste n'avaient pas de démarches préconisées à l'issue de la VPR.

- «\% dans dempreco»: $31 \%$ des salariés n'ayant pas de démarches préconisées à l'issue de la

VPR étaient déclarées aptes.

\begin{tabular}{|c|c|c|c|c|c|c|c|c|c|c|c|}
\hline & & & \multicolumn{2}{|c|}{$\begin{array}{l}\text { Démarches } \\
\text { préconisées } \\
\text { (dempreco) }\end{array}$} & \multicolumn{2}{|c|}{$\begin{array}{c}\text { Contact externe } \\
\text { à l'entreprise } \\
\text { (context) }\end{array}$} & \multicolumn{2}{|c|}{$\begin{array}{l}\text { Contact avec } \\
\text { l'employeur } \\
\text { (contactemp) } \\
\end{array}$} & \multicolumn{3}{|c|}{$\begin{array}{l}\text { Type de Pathologie } \\
\text { (typepatho) }\end{array}$} \\
\hline & & & NON & OUI & NON & OUI & NON & OUI & TMS & PSY & Autres \\
\hline \multirow{12}{*}{$\begin{array}{l}\text { Conclusion } \\
\text { De la visite } \\
\text { de reprise } \\
\text { (conclu) }\end{array}$} & \multirow{3}{*}{$\begin{array}{l}\text { Apte au } \\
\text { même poste }\end{array}$} & Effectif & 9 & 58 & 12 & 5 & 12 & 5 & 5 & 10 & 2 \\
\hline & & $\begin{array}{l}\% \text { dans } \\
\text { Conclu }\end{array}$ & $52,9 \%$ & $47,1 \%$ & $70,6 \%$ & $29,4 \%$ & $70,6 \%$ & $29,4 \%$ & $29,4 \%$ & $58,8 \%$ & $11,8 \%$ \\
\hline & & $\begin{array}{l}\% \text { dans } \\
\text { dempreco }\end{array}$ & $31,0 \%$ & $9,2 \%$ & $22,2 \%$ & $8,1 \%$ & $26,7 \%$ & $7,4 \%$ & $8,3 \%$ & $26,3 \%$ & $11,1 \%$ \\
\hline & \multirow{3}{*}{$\begin{array}{l}\text { Apte avec } \\
\text { aménagement }\end{array}$} & Effectif & 11 & 47 & 31 & 27 & 16 & 39 & 33 & 12 & 13 \\
\hline & & $\begin{array}{l}\% \text { dans } \\
\text { Conclu }\end{array}$ & $19,0 \%$ & $81,0 \%$ & $53,4 \%$ & $46,6 \%$ & $29,1 \%$ & $70,9 \%$ & $56,9 \%$ & $20,7 \%$ & $22,4 \%$ \\
\hline & & $\begin{array}{l}\% \text { dans } \\
\text { dempreco }\end{array}$ & $37,9 \%$ & $54,0 \%$ & $57,4 \%$ & $43,5 \%$ & $35,6 \%$ & $57,4 \%$ & $55,0 \%$ & $31,6 \%$ & $72,2 \%$ \\
\hline & \multirow{3}{*}{$\begin{array}{l}\text { Ne peut } \\
\text { occuper son } \\
\text { poste }\end{array}$} & Effectif & 0 & 1 & 1 & 0 & 0 & 1 & 1 & 0 & 0 \\
\hline & & $\begin{array}{l}\% \text { dans } \\
\text { Conclu } \\
\end{array}$ & $0,0 \%$ & $100,0 \%$ & $100,0 \%$ & $0,0 \%$ & $0,0 \%$ & $100,0 \%$ & $100,0 \%$ & $0,0 \%$ & $0,0 \%$ \\
\hline & & $\begin{array}{l}\% \text { dans } \\
\text { dempreco }\end{array}$ & $0,0 \%$ & $1,1 \%$ & $1,9 \%$ & $0,0 \%$ & $0,0 \%$ & $1,5 \%$ & $1,7 \%$ & $0,0 \%$ & $0,0 \%$ \\
\hline & \multirow{3}{*}{ Inapte } & Effectif & 9 & 31 & 10 & 30 & 17 & 23 & 21 & 16 & 3 \\
\hline & & $\begin{array}{l}\% \text { dans } \\
\text { Conclu }\end{array}$ & $22,5 \%$ & $77,5 \%$ & $25,0 \%$ & $75,0 \%$ & $42,5 \%$ & $57,5 \%$ & $52,5 \%$ & $40,0 \%$ & $7,5 \%$ \\
\hline & & $\begin{array}{l}\% \text { dans } \\
\text { dempreco }\end{array}$ & $31,0 \%$ & $35,6 \%$ & $18,5 \%$ & $48,4 \%$ & $37,8 \%$ & $33,8 \%$ & $35,0 \%$ & $42,1 \%$ & $16,7 \%$ \\
\hline \multirow{4}{*}{ Total } & & Effectif & 29 & 87 & 54 & 62 & 45 & 68 & 60 & 38 & 18 \\
\hline & & $\begin{array}{l}\% \text { dans } \\
\text { Conclu }\end{array}$ & $25,0 \%$ & $75,0 \%$ & $46,6 \%$ & $53,4 \%$ & $39,8 \%$ & $60,2 \%$ & $51,7 \%$ & $32,8 \%$ & $15,5 \%$ \\
\hline & & $\begin{array}{l}\text { \% dans } \\
\text { dempreco }\end{array}$ & $100,0 \%$ & $100,0 \%$ & $100,0 \%$ & $100,0 \%$ & $100,0 \%$ & $100,0 \%$ & $100,0 \%$ & $100,0 \%$ & $100,0 \%$ \\
\hline & & & \multicolumn{2}{|c|}{$\mathrm{p}=0,034$} & \multicolumn{2}{|c|}{$\mathrm{p}=0,03$} & \multicolumn{2}{|c|}{$\mathrm{p}=0,017$} & \multicolumn{3}{|c|}{$\mathrm{p}=0,04$} \\
\hline
\end{tabular}

